\title{
Structural analysis of the Toc75 Potra domains from Pisum sativum Karthik Srinivasan ${ }^{1}$, Nicholas Noinaj ${ }^{2}$, Srinivas Chakravarthy ${ }^{3}$, Satchal Erramilli ${ }^{4}$, Adrian Gonzalez ${ }^{5}$ \\ ${ }^{1}$ Purdue University ${ }^{2}$ Purdue University, ${ }^{3}$ BioCAT (Sector 18, APS), Illinois Institute of Technology, ${ }^{4}$ The University of Chicago, ${ }^{5}$ Molecular Templates Incorporated sriniv67@purdue.edu
}

Protein translocation across the chloroplast outer membrane is essential for photosynthesis in all green plants. This is because most chloroplast proteins (over 90\%) are encoded in the nucleus, translated in the cytoplasm, and must be imported into the chloroplasts to perform their functions. The translocon on the outer chloroplast membrane (TOC) complex orchestrates this vital translocation process and consists of three components: Toc75, Toc33/34 and Toc159 with unknown stoichiometries. Our lab seeks to elucidate the structural architecture of the TOC complex to gain mechanistic insights into protein translocation in chloroplasts. Toc75 is a $\beta$-barrel membrane protein that forms the channel of the TOC translocon and our lab has previously reported a crystal structure of the N-terminal polypeptide transport-associated (POTRA) domains from Arabidopsis thaliana. In this work, we demonstrate the generation of antigen-binding fragments (Fabs) that specifically recognize the POTRA domains from Pisum sativum (pea plant). Further, we report a $2 \AA$ crystal structure of pea Potra domain 2 in complex with a Fab. We also propose a homology model for Potra domains 1 and 3 and validate this hybrid model using size exclusion chromatography coupled with small angle X-ray scattering (SEC-SAXS). Future directions include screening more Fabs to obtain structures of all three Potra domains and probing for chaperone-like activity which has been demonstrated for the Arabidopsis variant. 